\title{
Spectrometric determination of clinically relevant fatty acids in the blood serum
}

\author{
A.V. Kalinin ${ }^{1, *}$, V.N. Krasheninnikov ${ }^{1}$, A.P. Sviridov², and V.N. Titov ${ }^{3}$ \\ ${ }^{1}$ Institute for Spectroscopy RAS, 108840 Troitsk, Moscow, Russia \\ ${ }^{2}$ Institute on Photon Technologies RAS, 108840 Troitsk, Moscow, Russia \\ ${ }^{3}$ Russian Cardiology Research and Production Complex, 119121 Moscow, Russia
}

\begin{abstract}
The content of fatty acid (FA) triglycerides in food and biological media is predicting traditionally using gas and liquid chromatographic methods. Named techniques aren't available for clinical labs due to their complexity. So, our objective was to develop the method and apparatus for rapid assay of a few clinically important FA as the saturated palmitic, mono unsaturated oleic and others in serum using near infrared spectrometer. As a result, the applicability of the FT spectrometer in the wavelength range of $0.9-1.8 \mu$ to analyze these FA in serum without sample preparation was confirmed. Besides, measurement specifications were determined and a correlations of the absorption spectra and contents of total triglycerides and cholesterol, palmitic, oleic, linoleic and arachidonic FA in serum were established
\end{abstract}

It is well known [1,2] that cardiovascular diseases and cancer - the main causes of human death, are statistically associated with an increased content of saturated fatty acid triglycerides (FA) compared to unsaturated FA in the blood. The most significant FA should include saturated palmitic $(\mathrm{C} 16: 0)$ and unsaturated oleic $(\mathrm{C} 18: 1)$ and linoleic (C18:2) FA. When treating cardiovascular diseases there is an urgent need for rapid methods of determining the content of these FA in blood. Traditional methods of FA analyses are the gas and liquid chromatography, they are of little use for express applications in clinical labs because of inefficacy, the complexity of sample preparation and high cost of analysis. The usage of near infrared spectroscopy, combined with the method of multi-component calibration - regression on projections to latent structures (PLS), seems promising for mass express-control of the above-mentioned FA in serum of patients [3].

Spectrometric analysis of FA triglycerides includes laborious calibration of the spectrometer on a huge number of standard samples with varying concentrations of several components. For the present study three sets of 27, 30 and 42 serum samples taken in different periods were selected from different patients of the clinical department of Russian Cardiology Research Complex (see. Table 1).

The content of total triglycerides (TG) and total cholesterol $(\mathrm{Ch})$ were measured in serum by enzymatic colorimetric techniques (GPO-PAP) Triglycerides -7743 Novo, with the liquid form of Miura, 250, and (CHOD-PAP) Cholesterol-7751 Novo and Miura, 250, respectively. The individual FA triglycerides content were quantifocated with extraction, separation and methylation of FA with the use of HPLC DIONEX Ultimate 3000 using

*Corresponding author: kalinin@isan.troitsk.ru 
Reprosil-Pur column C18-AQ 3 micron filler $150 \times 4.6 \mathrm{~mm}$. The mass spectrometer ABSCIEX QTRAP 5500 was used for registration of positively charged ions.

Table 1. Parameters of calibration sets of serum samples.

\begin{tabular}{|c|c|c|c|c|c|}
\hline $\begin{array}{c}\text { Set number } \\
\text { (sample quantity) }\end{array}$ & Analyt, unit & Min. & Max. & Average & SD \\
\hline \multirow[b]{2}{*}{$1(27)$} & $\mathrm{T \Gamma}, \mathrm{mmol} / 1$ & 0.33 & 8.83 & 2.3 & 1.3 \\
\hline & $\mathrm{X}, \mathrm{mmol} / \mathrm{l}$ & 0.07 & 7.12 & 3.8 & 1.4 \\
\hline \multirow{5}{*}{$2(30)$} & $\mathrm{T \Gamma}, \mathrm{mmol} / \mathrm{l}$ & 0.48 & 5.88 & 1.85 & 1.2 \\
\hline & $\mathrm{X}, \mathrm{mmol} / \mathrm{l}$ & 3.0 & 7.16 & 4.51 & 1.17 \\
\hline & $\mathrm{C} 16: 0, \mu \mathrm{g} / 1$ & 480 & 5880 & 1123 & 712 \\
\hline & $\mathrm{C} 18: 1, \mu \mathrm{g} / 1$ & 320 & 5544 & 839 & 201 \\
\hline & $\mathrm{C} 18: 2, \mu \mathrm{g} / 1$ & 442 & 6421 & 1021 & 266 \\
\hline \multirow{4}{*}{$3(42)$} & $\mathrm{C} 16: 0, \mu \mathrm{g} / 1$ & 305 & 5277 & 961 & 797 \\
\hline & $\mathrm{C} 18: 1, \mu \mathrm{g} / 1$ & 253 & 5544 & 878 & 821 \\
\hline & $\mathrm{C} 18: 2, \mu \mathrm{g} / 1$ & 419 & 6421 & 1119 & 949 \\
\hline & $\mathrm{C} 20: 4, \mu \mathrm{g} / 1$ & 96 & 467 & 228 & 98 \\
\hline
\end{tabular}

NIR spectrometry of FA triglyceride content in the serum is difficult because of dominating water absorption. Earlear, we found it possible to determine the individual FA in fat-oil mixtures with a small $(6 \%)$ level of water using the overtones and combinations of fundamental absorption bands near wavelengths of $1.15,1.4$ and $1.7 \mu$, for which the last band is about 4 times greater than the first [4]. To isolate the FA absorption against the background of a significant water uptake, we used the coincidence of fat absorption peak, and the relative failure of the water absorption near $1.7 \mu$ (Fig.1, a). The absorption spectra of serum samples in glass tube of $6.5 \mathrm{~mm}$ diameter were measured using MPA-FTNIR spectrometer, Bruker, Germany, at wavelength range $0.9-1.8 \mu$, during 4 minutes of accumulation time (64 scans), and the sample temperature of $36^{\circ} \mathrm{C}$, and then smoothed by Savitsky-Goley algorithm and differentiated - see. Figure 1, b).

The spectra of optical density $\mathrm{D}=\log \mathrm{I}_{0} / \mathrm{I}_{\mathrm{t}}$, where $\mathrm{I}_{0}$ - the flux across the empty cell, and $\mathrm{I}_{\mathrm{t}}$ - the flux passed through the sample in the spectral range of $0.9-1.8 \mu$, with the values of mass fraction of TG, Ch, and C16: 0, C18: 1 and C18: 2 FA in each of the three sets of samples were accumulated using PLS regression program ISCAP [3] for building the PLS models. Efficiency of them was evaluated by "leave-one-out" cross validation technique in accordance with [5] by the values of multiple correlation coefficient $r^{2}$ cv (reliability) and standard deviation SE from arbitral data. Next, we have tested each model on serum samples from two other sets, which were not used for calibration.

Selected by the testing, the best calibration models for a range of $0.9-1.8 \mu$ have shown the value of the standard error SE $10.8 \%$ and $9.3 \%$ for $\mathrm{TG}$ and $\mathrm{Ch}$, respectively, see Table 2. The variation registered in spectra of serum allowed to estimate the correlation with the mass fraction of TG, Ch, and of FA triglycerides: C16: 0, C18: 1, C18: 2 and C20: 4. As seen from Table 2, the models to determine the C16:0, C18:1 and C18:2 FA had shown a clear correlation of spectra with FA content at a value of multiple regression coefficient $r^{2}$ to within $0.82-0.97$. With the spectra of $3 r d$ set we have built a PLC models for the content of arachidonic C20: 4 FA. Check of its parameters by "leave-one-out" cross validation technique shows the values $\mathrm{n}=8, \mathrm{r}_{\mathrm{cv}}=0.68, \mathrm{SE}=15 \%$, which indicates the correlation, but requires testing on samples that are not included in the calibration set.

Besides PLC models to predict the content of C16: 0, C18: 1 and C18: 2 FA, the models were built to estimate the parameters of PLS regression on the content of cholesterol, which may "prevent" prediction of other FA. It is known [4], the parameter of PLC regression the vector of regression coefficients, (See Fig. 1c) allows one to select ranges of the spectra, which contain important data for determination of each FA and prevent the overlaping with the spectral ranges important to identify other components. For assessing the possible impact on the determination of oleic acid we have used the results of assignment of overtones and combinations in the NIR absorption spectra to the vibrations of $\mathrm{C}=\mathrm{C}$ and $\mathrm{C}$ - 
$\mathrm{H}$ bonds for oleic acid according to [6]. Thus obtained models GSDer and 2GSDer on 3-d set samples demonstrate the improved efficiency and accuracy for C18: 1 prediction.

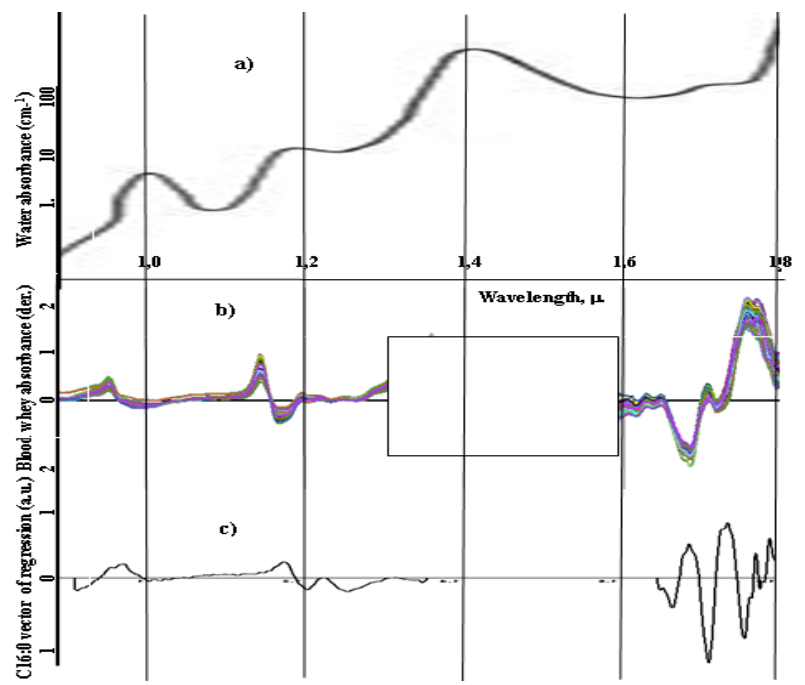

Fig. 1. Spectra: a) water absorptance (in logarithmic scale), b) differentiated absorbance of 27 serum samples, the noisy spectra - in frame, c) vector of regression coefficients for C18:1 FA.

Table 2. Parameters of prediction of TG, $\mathrm{Ch}$ and major FA content in independent serum samples: $\mathrm{K}$ is the number of calibration samples; GSDer and 2GSDer - smoothing and differentiating of the spectrum once or twice, $\mathrm{Rb}$ - removing spectra baseline, $\mathrm{n}$ - the number of latent variables; $\mathrm{SE}$ standard error, $\% ; \mathrm{r}_{\mathrm{p}}^{2}-$ multiple regression coefficient

\begin{tabular}{|c|c|c|c|c|c|c|c|c|c|c|c|c|c|c|c|c|}
\hline \multirow{2}{*}{$\begin{array}{l}\text { Set № } \\
(\mathrm{K})\end{array}$} & \multirow{2}{*}{$\begin{array}{l}\text { Spectra } \\
\text { treatment }\end{array}$} & \multicolumn{3}{|c|}{ TG } & \multicolumn{3}{|c|}{$\mathrm{Ch}$} & \multicolumn{3}{|c|}{ C16:0 } & \multicolumn{3}{|c|}{ C18:1 } & \multicolumn{3}{|c|}{ C18:2 } \\
\hline & & $\mathrm{n}$ & $r^{2} p$ & $\overline{\mathrm{SE}}$ & $n$ & $r^{2} p$ & SE & $n$ & $r^{2} p$ & SE & $n$ & $r_{p}^{2}$ & $\mathrm{SE}$ & $\mathrm{n}$ & $r^{2} p$ & SE \\
\hline $1(27)$ & GSDer & 9 & 0.97 & 11.0 & 4 & 0.86 & 10.9 & & & & & & & & & \\
\hline \multirow[t]{2}{*}{$2(30)$} & GSDer & 10 & 0.83 & 12.6 & 11 & 0.79 & 11.3 & 9 & 0.86 & 17 & 7 & 0.84 & 26 & 8 & 0.83 & 17 \\
\hline & 2GSDer & 9 & 0.96 & 10.8 & 4 & 0.88 & 9.3 & 8 & 0.87 & 18 & 7 & 0.86 & 26 & 8 & 0.85 & 18 \\
\hline \multirow[t]{3}{*}{$3(42)$} & $\mathrm{Rb}$ & 9 & 0.80 & 12.5 & 6 & 0.82 & 13.1 & 6 & 0.91 & 15 & 7 & 0.91 & 22 & 7 & 0.93 & 15 \\
\hline & GSDer & 7 & 0.86 & 12.3 & 4 & 0.88 & 12.2 & 2 & 0.93 & 12 & 2 & 0.91 & 22 & 4 & 0.93 & 14 \\
\hline & 2GSDer & 7 & 0.91 & 12.4 & 5 & 0.87 & 12.2 & 2 & 0.92 & 13 & 2 & 0.91 & 24 & 2 & 0.91 & 18 \\
\hline
\end{tabular}

In connection with the new concepts of the pathogenesis of atherosclerosis [7] the special diagnostic importance has the ratio of the FA C16:0 / C18:1 and C16:0 / C18:2 in the blood plasma. In the present work it was shown that these ratio $\mathrm{C} 16: 0 / \mathrm{C} 18: 1$ can be quickly evaluated using NIR spectrometer in the wavelength range 0.9-1.8 $\mu$. The prospect in this problem is to test the applicability of portable NIR spectrometer based on a linier InGaAs array photosensor of named range.

The work supported by the Russian Foundation for Basic Research, gr. № 16-02-00277.

\section{References}

1. V.N. Titov et al., Klinicheskaya Laboratornaya Diagnostika 7, 3 (2012)

2. http://umm.edu/health/medical/altmed/supplement/omega3-fatty-acids

3. A. Kalinin et al., J. Near Infrared Spectrosc. 16, 343 (2008)

4. A.V. Kalinin et al., Klinicheskaya Laboratornaya Diagnostika 11, 13 (2015)

5. S. Wold, M. Sjostrom, L. Eriksson, Chem. Intelligent. Lab. Syst. 58, 109 (2001)

6. F. Westad, A. Schmidt, M. Kermit, J. Near Infrared Spectrosc. 16, 265 (2008)

7. V.N. Titov, Pathogenesis 11, 18 (2013) 